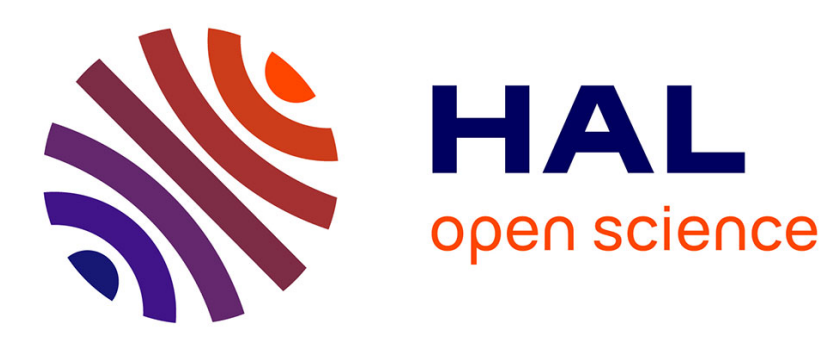

\title{
Zero-field magnetic resonance of cobalt ion pairs in $\mathrm{ZnO}$ nanocrystals \\ D Marin, S Tiwari, Sylvain Bertaina, Adrien Savoyant
}

\section{To cite this version:}

D Marin, S Tiwari, Sylvain Bertaina, Adrien Savoyant. Zero-field magnetic resonance of cobalt ion pairs in ZnO nanocrystals. Physical Review B, 2022, 105 (3), 10.1103/physrevb.105.035424 . hal03586794

\section{HAL Id: hal-03586794 \\ https://hal.science/hal-03586794}

Submitted on 24 Feb 2022

HAL is a multi-disciplinary open access archive for the deposit and dissemination of scientific research documents, whether they are published or not. The documents may come from teaching and research institutions in France or abroad, or from public or private research centers.
L'archive ouverte pluridisciplinaire HAL, est destinée au dépôt et à la diffusion de documents scientifiques de niveau recherche, publiés ou non, émanant des établissements d'enseignement et de recherche français ou étrangers, des laboratoires publics ou privés. 


\title{
Zero-field magnetic resonance of cobalt ion pairs in $\mathrm{ZnO}$ nanocrystals
}

\author{
D. Marin $\odot,{ }^{1}$ S. K. Tiwari $\odot,{ }^{2}$ S. Bertaina $\odot,{ }^{1}$ and A. Savoyant $\odot^{1}$ \\ ${ }^{1}$ Aix-Marseille Université, CNRS, IM2NP UMR 7334, 13397 Marseille Cedex 20, France \\ ${ }^{2}$ Department of Physics and Materials Science, Jaypee University of Information Technology, Waknaghat Solan, \\ Himachal Pradesh 173234, India
}

(Received 9 September 2021; revised 8 November 2021; accepted 6 January 2022; published 21 January 2022)

\begin{abstract}
Cobalt-doped $\mathrm{ZnO}$ nanoparticles (NPs) with different Co concentrations are investigated by means of $X$ and $Q$-band electron spin resonance (ESR) near liquid-helium temperature in both parallel and perpendicular modes. The high crystal quality of the NPs allows for the hyperfine-structure resolution within the single $\mathrm{Co}^{2+}$ ions' ESR powder spectra. Depending on cobalt concentration, common additional weak ESR lines are detected which are here demonstrated to arise from some $\mathrm{Co}^{2+}$ high-spin pairs with a distance of about 4-6 A. ESR simulations show that these $3 / 2$ spin pairs are weakly coupled by an isotropic Heisenberg Hamiltonian with either ferromagnetic or antiferromagnetic $J$ coupling constants, almost identical to those previously detected in bulk and microwire $\mathrm{ZnO}$ :Co. The presence of substantial (axial) single-ion anisotropy in $\mathrm{ZnO}$ :Co makes the different pairs' resonance positions strongly depending on the $J$ value. For resonance frequency $v$ in the microwave range, four cobalt pairs can satisfy the condition $|J| \sim h v / 3$ to resonate at almost zero magnetic field. Such near-zero-field transitions notably resonate in the parallel ESR mode, which is the signature of the gapped nonlinear Zeeman effect, which is of particular interest for highly stable atomic-clock transitions.
\end{abstract}

DOI: 10.1103/PhysRevB.105.035424

\section{INTRODUCTION}

Isolated spatially localized spins in semiconducting or insulating materials are promising quantum states for implementing information storage and processing technologies $[1,2]$. While current ultimate memory elements are still made of a huge number of spins or charges, reducing this number to unity or so has now became a reachable goal [3-5], leading to solotronic devices [6]. In addition, solid-state quantum computing and quantum metrology are also demanding for localized spin systems, embedded in a solid and robust insulating crystalline matrix $[7,8]$. The manipulation and detection of such spin systems are usually performed by optical pumping and various microwave pulses sequences $[9,10]$. In any case, the challenge is to overcome the inherent thermal and quantum fluctuations of these microscopically localized states and to achieve monitoring them by external physical probes. For such purposes, transition-metal (TM) doped semiconducting crystals have for a long time been recognized as relevant candidates. Depending on the site symmetry and $3 d$-electron number, these embedded TMs can bear a local electronic high-spin state lying within the energy band gap below the Fermi level [11-13]. The required temperature for observing such states mainly depends on the spin-lattice relaxation, for which embedded manganese and cobalt ions offer weak- and strong-coupling examples, respectively, resulting in high and low working temperatures. Also directly related to the spinlattice coupling, the single-ion magnetic anisotropy is very weak for manganese-doped compounds and notably high for cobalt-doped compounds.

While single-spin states of paramagnetic extrinsic impurities are commonly observed by electron spin resonance
(ESR), the pairing of these spin states, each one localized on distinct impurities, is more difficult to observe, in particular in very small objects such as nanoparticles (NPs). The main reasons are the low quantities of these pairs, the weakness of their coupling, the difficulty in their number control, and the ESR linewidth increase. This latter point is often due to the reduced crystallinity of nanostructures and to the dipolar broadening, which occurs even at relatively low impurity concentrations $[14,15]$. Nevertheless, these paired spins are still very interesting because of their richer state space compared to their single-spin counterparts $[16,17]$. If subjected to an important single-ion magnetic anisotropy, these spin pairs have a resonance line position highly dependent on isotropic Heisenberg $J$ coupling, which can occur at or near zero magnetic field. The combination of Heisenberg coupling and single-ion anisotropy can thus open a zero-field gap between resonating states $[18,19]$. For a static magnetic field applied perpendicular to the principal symmetry axis, the energy of the gapped resonating states has parabolic $\mathbf{B}$ field dependence with zero derivative at $B=0$, as required, in particular, for stable atomic-clock transitions [20].

In this context, cobalt-doped zinc oxide $(\mathrm{ZnO})$ appears to be an interesting material because it has been demonstrated that in the bulk, thin films, and microwires, aside from the single cobalt impurity signal, up to 11 distinct cobalt pairs can be separately detected by ESR, with weak $J$ coupling ranging from $25.6 \mathrm{~K}\left(17.8 \mathrm{~cm}^{-1}\right)$ for the nearest-neighbors pair to $0.008 \mathrm{~K}\left(0.0056 \mathrm{~cm}^{-1}\right)$ for the more distant one [14,2123]. Interestingly, some of these cobalt pairs have zero-field resonance in the microwave range, with potential applications in quantum technologies [24]. However, these Co pairs have been studied by ESR only with the magnetic field along the $c$ 
wurtzite axis, thus missing the gapped zero-field resonances. In addition, the question of these pairs' detection in nanosized $\mathrm{ZnO}$ :Co materials, where interesting confinement and electrostatic effects can occur and which are susceptible to being individually manipulated, can be raised.

In this paper, we present ESR results recorded for $\mathrm{ZnO}: \mathrm{Co}$ NPs near liquid-helium temperature in $X$ and $Q$ bands for perpendicular and parallel transition modes. The very high crystallinity of the studied NPs is highlighted by the hyperfine-coupling resolution of the single-ion spectrum. Some commonly observed weak ESR lines in ZnO:Co NP powder are here proven to arise from cobalt ion pairs, based on the analytical exact resolution of the zero-field Hamiltonian and the subsequent perturbation treatment of Zeeman interaction. This is then numerically confirmed by ESR spectra simulations in $X$ and $Q$ bands. The isotropic $J$ couplings between the localized $S=3 / 2$ spins are almost identical to those of bulk $\mathrm{ZnO}$, and four of them have zero-field resonance in the microwave range between 8 and $30 \mathrm{GHz}$. These different and distinct pair systems occurring in $\mathrm{ZnO}$ :Co NPs allow for the excitation of various zero-field atomic-clock transitions in the microwave range, possibly simultaneously addressed within a single nano-object.

\section{EXPERIMENT}

CoO-doped $\mathrm{ZnO}$ was prepared using the solid-state reaction method. Appropriate amounts of materials were carefully mixed and minced in a mortar. The powder was then annealed using the conventional method at $1100{ }^{\circ} \mathrm{C}$ for $2 \mathrm{~h}$. The $\mathrm{CoO}$ doping was done for $1 \%, 2 \%, 4 \%, 6 \%, 8 \%$, and $10 \%$. These are nominal concentrations, not the actual resulting concentration of the substitutional cobalt ion after the sample preparation. The resulting samples consist of microsized aggregates of nanoparticles, roughly equivalent to a polycrystalline material made of nanometric grains. For structural characterization $\mathrm{x}$-ray diffraction (XRD) was employed [25], which did not show secondary peaks related to Co clusters, other metal oxide inclusions, or impurities. Average NP size was calculated using the Scherrer formula and the XRD-obtained wurtzite lattice parameters $a$ and $c$. This mean diameter was found to be about $70 \mathrm{~nm}$, consistent with scanning electron microscope imaging [25]. It was observed that the lattice parameters $a$ and $c$ first decreased for increasing concentration up to $2 \%$ and then increased above $2 \%$ doping. The minimal change exhibited in the $a$ and $c$ parameters proves that Co ions are, indeed, situated at the tetrahedral site of the wurtzite structure.

$X$-band ESR experiments were performed on a conventional Bruker EMX spectrometer equipped with a standard double-mode (perpendicular and parallel) cavity $\left(v_{\perp}=\right.$ $9.629 \mathrm{GHz}$ and $v_{\|}=9.399 \mathrm{GHz}$ ), and $Q$-band experiments were performed on a conventional Bruker ELEXSYS spectrometer $\left(v_{Q}=33.971 \mathrm{GHz}\right)$. In both cases, the maximum available microwave power is $P_{0}=200 \mathrm{~mW}$, and the actual power $P$ reaching the sample is set by an attenuator $A$ defined by $A=10 \times \log _{10}\left(P_{0} / P\right)$.

Throughout the paper, care must be taken not to confuse the relative orientation of the static $(\mathbf{B})$ and microwave $\left(\mathbf{B}_{1}\right)$ magnetic fields (i.e., the parallel and perpendicular modes)

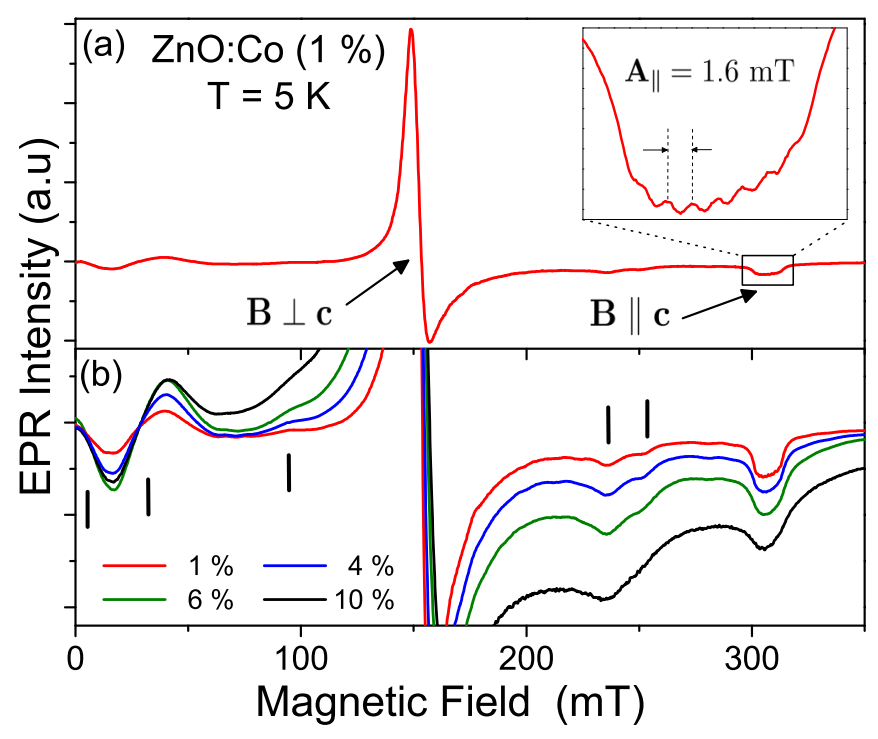

FIG. 1. $X$-band perpendicular mode ESR powder spectra of Codoped $\mathrm{ZnO}$ nanoparticles recorded at $T=5 \mathrm{~K}$ (a) for $1 \% \mathrm{Co}$ and (b) for $1 \%$ to $10 \%$ Co normalized to the perpendicular single-ion line. The inset of (a) is the $B \| c$ orientation magnification, showing the hyperfine coupling resolution $\left(A_{\|}=1.6 \mathrm{mT}\right)$. Black marks in (b) indicate the weak ESR lines not arising from single $\mathrm{Co}^{2+}$.

and the static field orientation with respect to the $c$ axis of the $\mathrm{ZnO}$ wurtzite structure. In the following, the sixfold $c$ axis of the wurtzite structure is along the $z$ laboratory direction, and the hexagonal plane lies in the $(x, y)$ laboratory plane.

\section{RESULTS}

\section{A. ESR experiments and modeling}

The $X$-band ESR powder spectrum of ZnO:Co NPs with the lowest cobalt doping (1\%) is shown in Fig. 1(a). The characteristic single $\mathrm{Co}^{2+} S=3 / 2$ powder spectrum with weak easy-plane axial anisotropy is observed [26-28], pointing to an efficient isovalent substitution of cobalt ions at cation sites $\left(\mathrm{Co}_{\mathrm{Zn}}\right)$. The weak easy-plane anisotropy favors an effective $\tilde{S}=1 / 2$ ground state, with effective $g$ factors $\tilde{g}_{\perp}=4.479$ $\left(B_{\perp}=153.5 \mathrm{mT}\right)$ and $\tilde{g}_{\|}=2.245\left(B_{\|}=306.3 \mathrm{mT}\right)$. For the B $\|$ c orientation, the hyperfine structure of the effective electronic $1 / 2$ spin with the $7 / 2$ spin of the ${ }^{59} \mathrm{Co}$ nucleus is resolved [ $A_{\|}=1.6 \mathrm{mT}$, Fig. 1(a) inset]. This value is identical to that of bulk $\mathrm{ZnO}: \mathrm{Co}[29,30]$, indicating a very similar cobalt environment in both cases since this coupling constant finely depends on the spatial extension of the Co $3 d$ wave functions. It also indicates the high crystallinity of the NPs under study, resulting in narrow linewidths $(<1.6 \mathrm{mT})$, which is observed quite rarely in $\mathrm{ZnO}$ :Co NP powder. As the temperature is increased above $\sim 30 \mathrm{~K}$, this single- $\mathrm{Co}^{2+}$ signal's intensity rapidly decreases because of the strong spin-lattice coupling of cobalt in $\mathrm{ZnO}$, which is responsible for fast relaxation [27,28]. In the $0 \%$ cobalt sample spectrum (not shown), only the usual core defect signal is observed at $351.6 \mathrm{mT}$ ( $g=1.96$ ), which disappears when cobalt is incorporated in the $\mathrm{ZnO}$ matrix, as previously reported in $\mathrm{ZnO}$ nanorods [31]. Besides this single- $\mathrm{Co}^{2+}$ signal, some other weak ESR lines are detected [black marks in Fig. 1(b)], previously seen in 


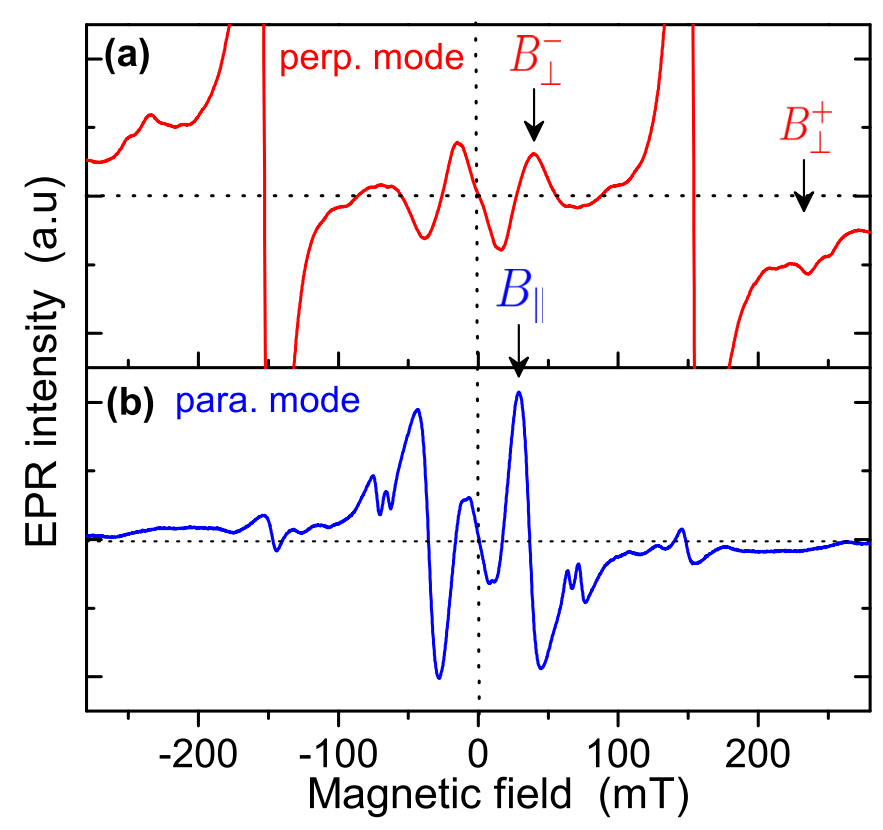

FIG. 2. (a) Perpendicular- and (b) parallel-mode $X$-band powder spectra of $\mathrm{ZnO}: \mathrm{Co}(2 \%)$ recorded at $T=10 \mathrm{~K}$ with magnetic field inversion.

several other ESR studies on ZnO:Co NP powder [32-34] but not analyzed or assigned to a specific center(s). In the following, these weak ESR lines are investigated and are demonstrated to arise from weakly coupled $\mathrm{Co}^{2+}-\mathrm{Co}^{2+}$ pairs with almost isotropic Heisenberg exchange coupling.

In order to examine the Co-concentration dependence, the various spectra in Fig. 1(b) were normalized to the maximum of the single-ion spectrum $(\mathbf{B} \perp \mathbf{c}$ orientation at $\sim 150 \mathrm{mT})$. Two effects are simultaneously observed: all linewidths are augmented, and the weak-line intensity increases up to an apparent saturation for the $10 \%$ Co sample. The first point is dipolar broadening, occurring when paramagnetic species concentration increases so that the resonance fields are more and more shifted around their normal position by small local fields. The second point is a clear indication that the observed weak lines under study are directly related to the cobalt concentration. This is consistent with the solubility limit of $\mathrm{Co}$ in $\mathrm{ZnO}$ occurring around $10 \% \mathrm{Co}$, where the crystal quality starts to become significantly degraded [35]. These results are very suggestive that the observed weak ESR lines are related to some complexes involving substitutional $\mathrm{Co}^{2+}$ ions. In Sec. III B, the spectra simulations will confirm that a weakly coupled cobalt-pair model correctly reproduces the experimental spectra with slightly modified exchange constants compared to bulk $\mathrm{ZnO}$ :Co.

A remarkable feature of the Co-related weak lines is that one of them apparently occurs at zero (or almost zero) magnetic field. This is clearly established by performing ESR experiments with a static magnetic field $\mathbf{B}$ inversion, moving from negative to positive values [Fig. 2(a)]. The zero-field line is then clearly visible, and the whole spectrum inversion symmetry with respect to the origin indicates a full paramagnetic system, with no significant ferromagnetic contributions.
In order to get deeper insight into these weak-field lines, the same experiment is performed in parallel mode, for which the single-ion resonance is suppressed. Parallel-mode ESR (microwave magnetic field $\mathbf{B}_{\mathbf{1}}$ along the static magnetic field $\mathbf{B}$ ) is quite rarely performed because the related transition probability is usually zero between levels displaying linear Zeeman splitting, which is forbidden by selection rules. However, these parallel-mode transitions can occur between levels separated by a gap, with a nonlinear Zeeman effect. The parallel-mode ESR experiment is reported in Fig. 2(b), which displays three notable features discussed and explained below. (1) The zero-field line is identically present in both modes, (2) the other weak-field line with a maximum at $B_{\perp}^{-}=39.6 \mathrm{mT}$ in the perpendicular mode has a maximum at $B_{\|}=28.8 \mathrm{mT}$ in the parallel mode (notation is explained below), and (3) a weak trace of the single-ion line persists at $B \sim 150 \mathrm{mT}$.

Point 3 is essentially due to a residual perpendicular-mode component of the microwave $\mathbf{B}_{\mathbf{1}}$ field. Actually, this field is totally parallel to $\mathbf{B}$ only at the very center of the ESR cavity, and since the sample has a certain spatial extension, part of it can still be excited by small perpendicular microwave components.

In the following, points 1 and 2 are qualitatively explained by considering a system of two weakly coupled $3 / 2$ spins subjected to easy-plane axial anisotropy. First, we note that among the 11 inequivalent pairs previously detected in $\mathrm{ZnO}$ :Co $[22,23]$, only 4 are relevant to be examined in our context, namely, those having a coupling constant in the microwave range $\left(J_{4}\right.$ to $\left.J_{7}\right)$. These four pairs fall within the weak-coupling case, that is, $|J|$ coupling being weaker than axial anisotropy $D$, and two of them have ferromagnetic coupling $(J>0)$.

The zero-field Hamiltonian for a given $\mathrm{Co}^{2+}$ pair consisting of two $3 / 2$ spins is

$$
H_{0}=D\left(S_{1 z}^{2}+S_{2 z}^{2}\right)-2 J \cdot \overrightarrow{S_{1}} \cdot \overrightarrow{S_{2}},
$$

including easy-plane single-ion anisotropy $(D=$ $\left.2.76 \mathrm{~cm}^{-1}=3.97 \mathrm{~K}\right)$ and isotropic Heisenberg coupling $(|J|=0.134$ to $0.382 \mathrm{~K})$. Since this Hamiltonian does not couple states with different total spin projections, it can be exactly solved within the whole 16-dimensional state space by separately diagonalizing submatrices with definite total spin projection [25]. Keeping in mind that $|J|<D$, the level diagram of Fig. 3(a) is obtained, for which only the lowest levels splittings are shown. In the antiferromagnetic case displayed in Fig. 3, the resulting ground state corresponds to an effective $\tilde{S}=0$ spin, and the first two excited states correspond to an effective anisotropic $\tilde{S}=1$ spin. Exact expressions of the zero-field splittings $\Delta_{1}$ and $\Delta_{2}$ are obtained, involving the square roots of the $D$ and $J$ polynomials [25]. However, the zero-field splitting of interest $\Delta_{1}$ is more conveniently expanded in powers of the $J / D$ ratio, up to second order,

$$
\Delta_{1}=3|J|\left(1-\frac{13 J}{4 D}+\frac{5 J^{2}}{2 D^{2}}\right),
$$

thus making apparent the limiting value $3|J|$ as $J / D \rightarrow 0$ and the important dependence on the coupling sign. For the four 


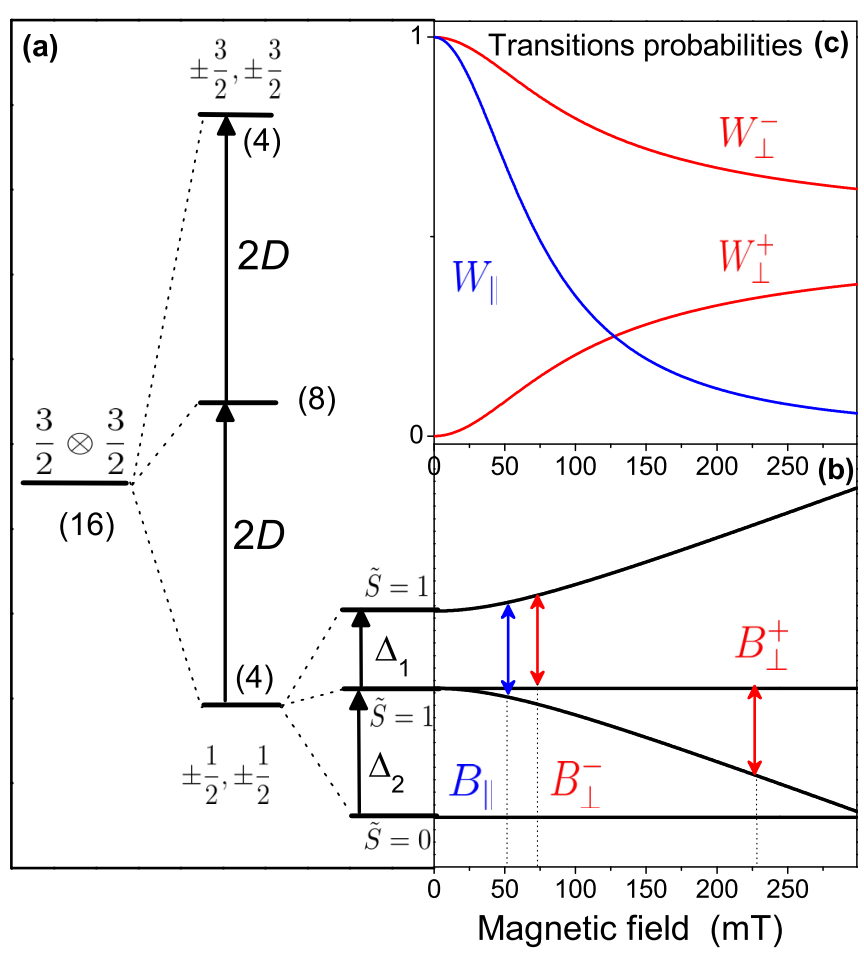

FIG. 3. (a) Zero-field level diagram for a cobalt pair under easyplane anisotropy $(D>0)$ and isotropic antiferromagnetic coupling $(J<0)$. (b) Magnetic field dependence of the lowest pair's levels showing perpendicular and parallel transitions for the $\mathbf{B} \perp \mathbf{c}$ orientation. (c) Normalized transition probabilities for the two perpendicular $\left(B_{1} \| y\right)$ and single parallel $\left(B_{1} \| x\right)$ transitions.

considered pairs, the $J / D$ ratio goes from 0.034 to 0.096 , so that the coupling-sign dependence in (2) cannot be neglected.

To explain the weak-field lines' position and intensity, the Zeeman interaction $H_{z}=\mu\left(\overrightarrow{S_{1}}+\overrightarrow{S_{2}}\right) \tilde{g} \vec{B}$ is added to the Hamiltonian (1), with $\mu$ being the Bohr magneton and $\tilde{g}$ being the $g$ factor tensor. Dealing with a powder of axially symmetric elements, the dominant orientations in the powder spectra are those for which $\mathbf{B} \perp c$. Consequently, the magnetic field orientation is chosen along the $x$ laboratory axis for the analytical treatment. The case where $\mathbf{B} \| c$ was treated elsewhere for microcrystals [23], which here gives much weaker lines at higher fields in the powder spectra [see Fig. 1(a)]. Since we are interested in the low-field lines, the Zeeman term can be treated as a perturbation behind $H_{0}$, restricting the state space to the four lowest-energy exact eigenstates of $H_{0}$. The first-order perturbation is zero, and the second-order perturbation leads to a parabolic magnetic field dependence for two of the four levels [see Fig. 3(b) and Supplemental Material [25] for details].

The three resonance field positions depicted in Fig. 3(b) (two in the perpendicular mode and one in the parallel mode) are given by the relations

$$
\begin{gathered}
h v_{\perp}=\Delta_{1}+K\left(B_{\perp}^{-}\right)^{2}, \\
h v_{\perp}=K\left(B_{\perp}^{+}\right)^{2}, \\
h v_{\|}=\Delta_{1}+2 K\left(B_{\|}\right)^{2},
\end{gathered}
$$

where $\Delta_{1}$ is approximatively given by Eq. (2) and the coefficient $K$ is obtained by the second-order perturbative treatment of the Zeeman term. As for $\Delta_{1}, K$ is much more conveniently expressed by a second-order approximation in $J / D$ :

$$
K=\frac{4 g_{\perp}^{2} \mu^{2}}{3|J|}\left(1+\frac{25 J}{4 D}+\frac{35 J^{2}}{16 D^{2}}\right) .
$$

For the perpendicular mode, the microwave field $B_{1}$ can lay along the $y$ or $z$ axis, leading to different transition probabilities at the same positions $B_{\perp}^{-}$and $B_{\perp}^{+}$. However, as $B$ goes to zero, the $B_{1} \| z$ polarization gives no ESR lines because of the zero probability of its low-field line (see [25] and discussion in Sec. IV). The three relevant transition probabilities are plotted in Fig. 3(c), for which we give here explicitly only that in the parallel mode for $J / D \rightarrow 0$ :

$$
W_{\|} \propto\left[1+\left(\frac{4 g_{\perp} \mu B}{3 J}\right)^{2}\right]^{-1} .
$$

The parallel-transition probability goes to zero as the $B$ field is increased, when the level slope becomes linear. For B $\| c$, there is no gap between the resonating levels, the Zeeman splitting is fully linear, and the parallel-mode transition probability is strictly zero for every field (see the level diagram in Ref. [23]).

From this simple pair model, all the qualitative features of both perpendicular and parallel modes spectra are explained. In the perpendicular mode two transitions are predicted (for each pair) at $B_{\perp}^{-}$and $B_{\perp}^{+}$, around the single-ion resonance field $(\sim 150 \mathrm{mT})$, just as experimentally observed in Fig. 2(a). The high-field line has a lower intensity than the low-field one, consistent with the transition probability calculation depicted in Fig. 3(c). It is also clear that if $\Delta_{1}>h v_{\perp}$, only the highfield line can be observed and that if $\Delta_{1} \ll h v_{\perp}$, the two lines finally fall into the single-ion linewidth, indeed contributing to the dipolar broadening.

In the parallel mode, only a low-field line occurs, which requires the very particular condition that $\Delta_{1} \lesssim h v_{\|}$. If not, either the resonance condition is never met, or the transition probability goes to zero. Note that at exactly zero magnetic field, the distinction between the parallel and perpendicular modes vanishes, so that the zero-field line is equally visible in both modes.

Finally, our analysis shows that a relation must exist between the low-field resonance position in the perpendicular and parallel modes. Assuming $v_{\perp}=v_{\|}$and that $J / D$ goes to zero in Eqs. (3) and (5), we obtain $B_{\perp}^{-} / B_{\|} \sim \sqrt{2}$. Experimentally, using the powder spectrum line maximum to determine $B_{\perp}^{-}$and $B_{\|}$, this ratio is found to be 1.38 (see Fig. 2 and point

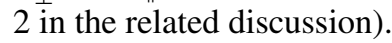

\section{B. Spectra simulations}

In order to go beyond the qualitative analysis made above and to confirm the cobalt-pair origin of the observed weak lines, ESR spectra simulations in both $X$ and $Q$ bands are performed with the MATLAB EASYSPIN toolbox [36]. The $16 \times$ 16 matrix of the Hamiltonian $H=H_{0}+H_{z}$ is numerically diagonalized for each $\mathbf{B}$ field value, beyond any perturbation scheme. The simulated spectra are constructed by 
TABLE I. Spin Hamiltonian parameters used in the spectra simulations in Fig. 5. The $w_{i}$ 's, $\alpha_{\perp}^{i}$ 's and $\alpha_{\|}^{i}$ 's, $E_{i}$ 's, and $J_{i}$ 's are, respectively, the pairs weight, the reduction factor for $\tilde{g}$ tensor components, the single-ion rhombic parameter, and the isotropic coupling constant for the $i$ th pair. Coupling constants are given in kelvins.

\begin{tabular}{cccccccc}
\hline \hline$i$ & $\alpha_{\perp}^{i}$ & $\alpha_{\|}^{i}$ & $E_{i}\left(\mathrm{~cm}^{-1}\right)$ & $w_{i}$ & $J_{i}(\mathrm{NP})^{\mathrm{a}}$ & $J_{i}(\mathrm{MW})^{\mathrm{b}}$ & $J_{i}(\mathrm{bulk})^{\mathrm{c}}$ \\
\hline 4 & 1.00 & 1.00 & 0.05 & 2 & -0.378 & -0.379 & -0.382 \\
5 & 0.90 & 0.90 & 0.04 & 6 & +0.351 & +0.363 & +0.347 \\
6 & 1.00 & 0.93 & 0.00 & 6 & +0.145 & +0.170 & +0.168 \\
7 & 1.00 & 0.94 & 0.00 & 3 & -0.137 & -0.138 & -0.134 \\
\hline \hline
\end{tabular}

a This work.

${ }^{\mathrm{b}}$ Reference [23].

${ }^{\mathrm{c}}$ Reference [22].

superimposing the single- $\mathrm{Co}^{2+}$ powder spectrum and those of the various $\mathrm{Co}^{2+}-\mathrm{Co}^{2+}$ pairs, each with its specific weight. The zero-field pair Hamiltonian is that of Eq. (1), except for the closest pairs (strongest couplings $J_{4}$ and $J_{5}$ ), for which a small rhombic term needs to be added (see below). The axial anisotropy parameter is set to its bulk value $\left(D=2.76 \mathrm{~cm}^{-1}\right)$ [37,38]. The axial $\tilde{g}$ tensor components $g_{\perp}$ and $g_{\|}$are first set to their single-ion bulk values $\left(g_{\perp}^{0}=2.277\right.$ and $\left.g_{\|}^{0}=2.238\right)$, and so are the isotropic Heisenberg coupling constants $J$ $[22,23]$. These last three parameters (for each pair) are then slightly adjusted in order to reproduce, at best, the various experimental spectra. The modifications of $g_{\perp}$ and $g_{\|}$are respectively defined by the reduction factors $\alpha_{\perp}=g_{\perp} / g_{\perp}^{0}$ and $\alpha_{\|}=g_{\|} / g_{\|}^{0}$.

Among all the previously detected pairs in bulk $\mathrm{ZnO}$ :Co, only four are relevant for the present simulations, namely, $J_{4}$ to $J_{7}$. Stronger coupled pairs $\left(J_{1}\right.$ to $\left.J_{3}\right)$ do not resonate at all in the $X$ - or $Q$-band magnetic field ranges (up to $1.4 \mathrm{~T}$ ), and weakly coupled pairs $\left(J_{8}\right.$ to $\left.J_{11}\right)$ resonate as satellite lines and fall within the single-ion linewidth. In each simulated spectrum, the four relevant pairs are included with the spin Hamiltonian parameters reported in Table I. In $X$-band spectra only $J_{6}$ and $J_{7}$ pairs give notable contributions, whereas in the $Q$-band spectrum only $J_{4}$ and $J_{5}$ do. For all simulations, the single-ion spectrum to pair spectra ratio is $170: w_{i}$, with $w_{i}$ being the relative weight of the $i$ th pair (Table I).

The $X$-band simulations are performed for both the perpendicular $\left(v_{\perp}=9.629 \mathrm{GHz}\right)$ and parallel $\left(v_{\|}=9.399 \mathrm{GHz}\right)$ modes [Figs. 4(a) and 4(b)]. These simulations, based on the parameters in Table I, display overall good agreement with the experimental spectra. The zero-field resonance line arises from the $J_{7}$ pair, while the weak-field line arises from $J_{6}$.

In order to strengthen the modeling, $Q$-band ESR $\left(v_{Q}=\right.$ $33.971 \mathrm{GHz}$ ) was performed on a $1 \%$ Co sample and then simulated. In this case, the spectrum is still dominated by the single-ion $\mathbf{B} \perp \mathbf{c}$ line $(\sim 560 \mathrm{mT})$, but weak ESR lines mainly arise from the $J_{4}$ and $J_{5}$ pairs, as demonstrated by the simulation (Fig. 5). Interestingly, a single-ion rhombic zero-field splitting contribution in the form

$$
H_{R}=E\left(S_{1 x}^{2}-S_{1 y}^{2}+S_{2 x}^{2}-S_{2 y}^{2}\right)
$$

must be added to the Hamiltonian (1) in order to correctly reproduce the experimental spectrum. Concretely, the rhombic $E$ parameter is responsible for the line splitting of the
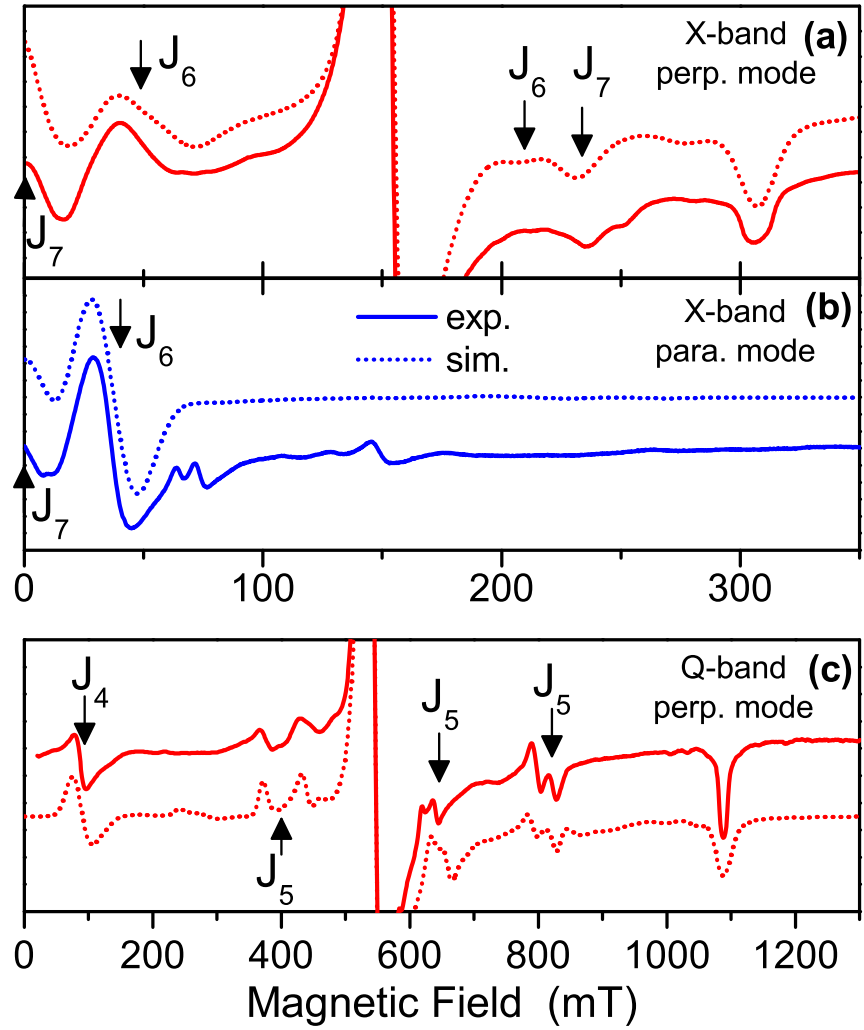

FIG. 4. Comparison between experimental 2\% Co (solid lines) and simulated (dotted lines) spectra. (a) Perpendicular-mode $X$ band, (b) parallel-mode $X$ band, and (c) perpendicular-mode $Q$ band.

ferromagnetic pair $J_{5}$ and a shift for the antiferromagnetic pair $J_{4}$ (Fig. 5). The values of these rhombic $E$ parameters are very weak $\left(0.04-0.05 \mathrm{~cm}^{-1}\right)$ compared to the axial parameter $\left(2.76 \mathrm{~cm}^{-1}\right)$. This indicates a very slight rhombic distortion, mutually caused by the $\mathrm{Co}^{2+}$ ions of the pair, that is logically

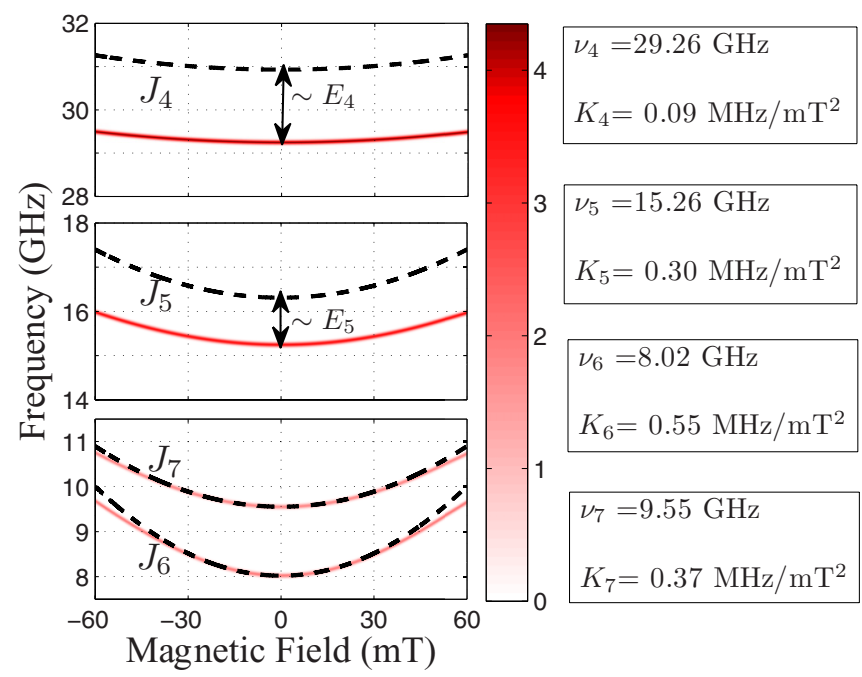

FIG. 5. Numerical color map of the powder ESR absorption intensity as a function of field and frequency in the perpendicular mode for $J_{4}$ to $J_{7} \mathrm{Co}^{2+}$ pairs. The dashed line is the analytical resonance field transition obtained from (3). 
stronger for the closest pairs. This rhombic distortion may exist for the $J_{6}$ and $J_{7}$ pairs with even weaker values. However, the importance of this distortion decreases with the $J / D$ ratio when the single-spin projections $\pm 1 / 2$ and $\pm 3 / 2$ tend to be completely decoupled in the ground multiplet. It thus cannot be detected in the presented $X$-band experiments, even if it is present for $J_{6}$ and $J_{7}$.

In the above-presented simulations some experimental features are not reproduced, for example, the weak double line at about $70 \mathrm{mT}$ in the $X$-band parallel mode. This can be accounted for (and, indeed, all simulations can be notably improved) by introducing new parameters such as anisotropic exchange couplings $\left(J_{x}, J_{y}, J_{z}\right)$, rhombic $\tilde{g}$ tensors, dipolar contributions, different $D$ and $E$ parameters for each spin of the pairs, and some other model refinements. However, this notably increases the parameters number and lowers the physical relevance of the model. Hence, without reaching a perfect spectrum simulation, we regard the pair's origin of the weak ESR lines to be as demonstrated above because almost all of the experimental features of the weak ESR lines are analytically and numerically reproduced consistently.

\section{DISCUSSION}

In the context of quantum technology applications, cobaltdoped $\mathrm{ZnO}$ materials appear to be interesting semiconducting materials since several stable and microscopically localized microwave transitions can occur within, at, or near zero magnetic field. For weak applied magnetic field (within the hexagonal plane, $\mathbf{B} \perp \mathbf{c}$ ), a parabolic dependence of the resonating levels is found, allowing for both parallel and perpendicular ESR transitions, the distinction of which vanishes as the applied static magnetic field goes to zero.

To experimentally determine the zero-field resonance frequencies, one could, in principle, scan the microwave frequency, searching for absorption maxima. Then, Eq. (2) would allow for the determination of the corresponding $J$ value, without any influence of the $\tilde{g}$ tensor and B field. However, in conventional ESR spectrometers the frequency is kept constant while the magnetic field is swept in turn. Therefore, the scheme must be inverted: we first determine the $J$ couplings using the field-swept experiment and related simulations, and after that, we compute the frequency-swept powder simulations, which we repeat for different $\mathbf{B}$ field values of interest. This procedure leads to a mapping of microwave absorption intensity as a function of frequency and static B field. Doing this for each pair with parameters taken from Table I, we obtain the exact frequency vs field dependence of the powder absorption intensity depicted in Fig. 5 for the perpendicular mode with a common linewidth of $80 \mathrm{MHz}$. It is found that the $J_{4}, J_{5}, J_{6}$, and $J_{7}$ pairs have zero-field resonance frequencies equal to $29.26,15.26,8.02$, and $9.55 \mathrm{GHz}$, respectively.

The other important parameter is the second derivative of these curves at $B=0$, which indicates the parabola's flatness near the origin. Contrary to the zero-field resonance frequency, this parameter is difficult to evaluate numerically from the map in Fig. 5. However, near zero magnetic field, the frequency vs field dependence is parabolic and can, in principle, be fitted by the perturbative expressions (3) and (5) with variable $B$ field. This leads to a value of $4 \mathrm{~K}$ in the parallel mode and $2 \mathrm{~K}$ in the perpendicular mode for the second derivative at the origin. The perpendicular mode thus appears to be more appropriate for stable clock transitions since the weakest possible curvature is searched for in this context.

In order to evaluate how accurate Eqs. (3) and (5) are, we plot them as dashed lines in the map in Fig. 5. For the weakest-coupled pairs $\left(J_{6}\right.$ and $\left.J_{7}\right)$ the perturbative expressions have good accuracy up to $\sim 40 \mathrm{mT}$, using the second-order expansions (2) and (6) for $\Delta_{1}$ and $K$, respectively. On the contrary, for $J_{4}$ and $J_{5}$, even an exact expression for $\Delta_{1}$ and the complete one for $K$ given in Supplemental Material do not lead to acceptable agreement between numerical and analytical results (Fig. 5). The reason is clearly that the simulations of these pair spectra require the addition of rhombic parameters $E_{4}$ and $E_{5}$, which are not included in the exact analytical expression of $\Delta_{1}$ or in the complete perturbative expression of $K$. However, the comparison between exact and perturbative results for $J_{4}$ and $J_{5}$ in Fig. 5 shows that this discrepancy is mainly a shift in frequency, of order $E_{4}$ and $E_{5}(\sim 1 \mathrm{GHz})$. This means that the rhombic correction significantly affects only the $\Delta_{1}$ parameters and does not affect $K$ very much. The $K$ parameter can then be used in the complete form given in the Supplemental Material to evaluate the second derivative near zero field for all of the four studied pairs.

Finally, we obtain the value of $K$ for each pair $\left(K_{4}, \ldots, K_{7}\right)$ from 0.09 to $0.55 \mathrm{MHz} / \mathrm{mT}^{2}$ (see Fig. 5). Qualitatively, we see that this second derivative near zero field is inversely proportional to $|J|$ and, for given $|J|$, is weaker for antiferromagnetic coupling $(J<0)$.

Regarding the pair location within the $\mathrm{ZnO}$ matrix, according to the classification given in Refs. [23,39], we can assume that the four $\mathrm{Co}^{2+}-\mathrm{Co}^{2+}$ pairs under study must have an inner distance from 4 to $6 \AA$. The presence of a rhombic correction for $J_{4}$ and $J_{5}$ moreover suggests that the corresponding pairs are certainly not "axial pairs"; that is, they are not oriented along the wurtzite $c$ axis. Clearly, making an exact and unequivocal correspondence between the ESR-detected couplings and the different classes of topological pairs is difficult, but in fact, it is not very necessary. Assuming that a given coupling corresponds to a given pair, making technological use of it can be considered. In this respect, it is interesting to note that the weakness of the detected couplings is such that they are significantly below the energetic resolution of $a b$ initio calculation $[40,41]$, pointing to the extreme sensitivity of ESR. This should be compared to effective magnetization measurements (e.g., by superconducting quantum interference device), in which only the weak paramagnetic contributions from the singles would be detectable.

Regarding the coupling mechanisms, as mentioned in Ref. [22], the ferromagnetic-like couplings $J_{5}$ and $J_{6}$ point to the presence of some carrier-mediated exchange contributions (besides the superexchange ones), which are probably related to the residual $n$-type conductivity of $\mathrm{ZnO}$. This kind of indirect exchange could certainly be stimulated by some interband external optical excitations.

Finally, the most limiting factors for the use of such paired spins in a quantum technology context is the difficulty controlling their number and nature. Basically, in slightly doped semiconducting crystals, isolated impurities are the most 
frequently occurring case, while bigger and bigger complexes of impurities (pairs, triplets, etc.) have lower and lower probabilities to occur. Obtaining a certain number of pairs a priori implies a relatively high impurity concentration and, consequently, a high number of singles. The main problem is then connected to the spin-spin relaxation: a high number of unpaired spins in the vicinity of a given spin-pair implies very short dephasing time after some microwave pulses sequence, resulting in an enlarged homogeneous linewidth (dipolar cross relaxation). This can prevent detection and manipulation of such a spin system by time-dependent microwave perturbation, favoring fast decoherence.

The situation, however, is susceptible to modification in the nanostructure context. In such cases, with the doping being certainly inhomogeneous, it is conceivable that some very small NPs a diameter of about twice the pair's distance will contain only one or a few pairs. Within an aggregate of NPs, these pairs would thus be somewhat shielded from the other singles' and pairs' impurities by each NP surface, preventing unwanted spin-spin relaxation and homogeneous broadening. Even if it is still hypothetical, such a scenario still points to the interesting potentiality of spin-pair physics in semiconducting nanocrystals. In this context of randomly oriented nanocrystals, it is worth mentioning that for zero static magnetic field the resonance frequency does not at all depend on individual element orientation but the resonance intensity does. In fact, for $B=0$, the transition probability is zero for a microwave linear polarization along the $z$ axis of the NP and nonzero for this polarization lying within the $(x, y)$ easy plane [25]. As a consequence, only those NPs with the $z$ axis perfectly aligned with the microwave $B_{1}$ field will not resonate at all, while the rest of them will contribute to the resonance, with higher contributions from those oriented more perpendicular to the microwave field.

\section{CONCLUSION}

Cobalt-doped ZnO NPs with a diameter of about $70 \mathrm{~nm}$ were investigated by ESR near the liquid-helium temperature in both the parallel and perpendicular modes. For a Co concentration ranging from $1 \%$ to $10 \%, X$ - and $Q$-band ESR allowed for the detection of four Co-Co pairs. These simple Co complexes were described by two axially anisotropic $3 / 2$ spins coupled by the isotropic Heisenberg interaction with either ferromagnetic and antiferromagnetic coupling constants ranging from $|0.137|$ to $|0.378| \mathrm{K}$. These couplings respectively correspond to zero-field microwave frequencies from 8 to $30 \mathrm{GHz}$, with low-curvature parabolic $B$ field dependence. Atomiclike clock transitions could then be addressed in a single and robust inorganic nanocrystal.

\section{ACKNOWLEDGMENTS}

The authors gratefully thank Dr. S. Grimaldi for helping in using the $Q$-band spectrometer of the Bioénergétique et Ingénierie des Protéines (BIP) laboratory (UMR 7281), Marseille, France, and Prof. R. Hayn for helpful discussions. This work is supported by Agence Nationale de la Recherche (ANR "SPIMAN", ANR-21-CE09-0027- 01). We further acknowledge financial support from the French Federation of Research RENARD (FR 3443).
[1] G. Balasubramanian, P. Neumann, D. Twitchen, M. Markham, R. Kolesov, N. Mizuochi, J. Isoya, J. Achard, J. Beck, J. Tissler, V. Jacques, P. R. Hemmer, F. Jelezko, and J. Wrachtrup, Nat. Mater. 8, 383 (2009).

[2] V. V. Dobrovitski, G. D. Fuchs, A. L. Falk, C. Santori, and D. D. Awschalom, Annu. Rev. Condens. Matter Phys. 4, 23 (2013).

[3] B. Kiraly, A. N. Rudenko, W. M. J. van Weerdenburg, D. Wegner, M. I. Katsnelson, and A. A. Khajetoorians, Nat. Commun. 9, 3904 (2018).

[4] V. Tiwari, M. Arino, S. Gupta, M. Morita, T. Inoue, D. Caliste, P. Pochet, H. Boukari, S. Kuroda, and L. Besombes, Phys. Rev. B 104, L041301 (2021).

[5] P. Koenraad and M. Flatté, Nat. Mater. 10, 91 (2011).

[6] J. Kobak, T. Smoleński, M. Goryca, M. Papaj, K. Gietka, A. Bogucki, M. Koperski, J.-G. Rousset, J. Suffczyński, E. Janik, M. Nawrocki, A. Golnik, P. Kossacki, and W. Pacuski, Nat. Commun. 5, 3191 (2014).

[7] G. Wolfowicz, A. M. Tyryshkin, R. E. George, H. Riemann, N. V. Abrosimov, P. Becker, H.-J. Pohl, M. L. W. Thewalt, S. A. Lyon, and J. J. L. Morton, Nat. Nanotechnol. 8, 561 (2013).

[8] J. Salfi, M. Tong, S. Rogge, and D. Culcer, Nanotechnology 27, 244001 (2016).

[9] D. E. Reiter, T. Kuhn, and V. M. Axt, Phys. Rev. B 85, 045308 (2012)

[10] L. Besombes and H. Boukari, Phys. Rev. B 89, 085315 (2014).

[11] N. Jedrecy, H. J. von Bardeleben, Y. Zheng, and J.-L. Cantin, Phys. Rev. B 69, 041308(R) (2004).
[12] T. Devillers, M. Rovezzi, N. G. Szwacki, S. Dobkowska, W. Stefanowicz, D. Sztenkiel, A. Grois, J. Suffczyński, A. NavarroQuezada, B. Faina, T. Li, P. Glatzel, F. d'Acapito, R. Jakieła, M. Sawicki, J. A. Majewski, T. Dietl, and A. Bonanni, Sci. Rep. 2, 722 (2012).

[13] T. Smoleński, T. Kazimierczuk, J. Kobak, M. Goryca, A. Golnik, P. Kossacki, and W. Pacuski, Nat. Commun. 7, 10484 (2016).

[14] P. Sati, A. Stepanov, and V. Pashchenko, Low Temp. Phys. 33, 927 (2007).

[15] A. Savoyant, H. Alnoor, S. Bertaina, O. Nur, and M. Willander, Nanotechnology 28, 035705 (2017).

[16] L. Besombes, C. L. Cao, S. Jamet, H. Boukari, and J. Fernàndez-Rossier, Phys. Rev B 86, 165306 (2012).

[17] O. Krebs and A. Lemaître, Phys. Rev. Lett. 111, 187401 (2013).

[18] P. Sati, R. Hayn, R. Kuzian, S. Régnier, S. Schäfer, A. Stepanov, C. Morhain, C. Deparis, M. Laügt, M. Goiran, and Z. Golacki, Phys. Rev. Lett. 96, 017203 (2006).

[19] J. C. Oberg, M. R. Calvo, F. Delgado, M. Moro-Lagares, D. Serrate, D. Jacob, J. Fernández-Rossier, and C. F. Hirjibehedin, Nat. Nanotechnol. 9, 64 (2014).

[20] M. Rubín-Osanz, F. Lambert, F. Shao, E. Rivière, R. Guillot, N. Suaud, N. Guihéry, D. Zueco, A.-L. Barra, T. Mallah, and F. Luis, Chem. Sci. 12, 5123 (2021).

[21] D. V. Azamat, A. Dejneka, V. A. Trepakov, L. Jastrabik, M. Fanciulli, V. Y. Ivanov, M. Godlewski, V. I. Sokolov, J. Rosa, and A. G. Badalyan, Phys. Status Solidi RRL 5, 138 (2011). 
[22] S. D'Ambrosio, V. Pashchenko, J.-M. Mignot, O. Ignatchik, R. O. Kuzian, A. Savoyant, Z. Golacki, K. Grasza, and A. Stepanov, Phys. Rev. B 86, 035202 (2012).

[23] A. Savoyant, F. Giovannelli, F. Delorme, and A. Stepanov, Semicond. Sci. Technol. 30, 075004 (2015).

[24] J. C. Bardin, D. H. Slichter, and D. J. Reilly, IEEE J. Microwaves 1, 403 (2021).

[25] See Supplemental Material at http://link.aps.org/supplemental/ 10.1103/PhysRevB.105.035424 for structural characterization and modeling details.

[26] J. Chess, G. Alanko, D. A. Tenne, Ch. B. Hanna, and A. Punnoose, J. Appl. Phys. 113, 17C302 (2013).

[27] F. Acosta-Humànez, R. Cogollo Pitalùa, and O. Almanza, J. Magn. Magn. Mater. 329, 39 (2013).

[28] T. J. Castro, P. A. M. Rodrigues, A. C. Oliveira, F. Nakagomi, J. Mantilla, J. A. H. Coaquira, A. Franco Jùnior, H. V. S. Pessoni, P. C. Morais, and S. W. da Silva, J. Appl. Phys. 121, 013904 (2017).

[29] T. L. Estle and M. De-Wit, Bull. Am. Phys. Soc. 6, 445 (1961).

[30] A. Hausmann, Phys. Status Solidi B 31, K131 (1969).
[31] A. Savoyant, H. Alnoor, O. Pilone, O. Nur, and M Willander, Nanotechnology 28, 285705 (2017).

[32] J. Hays, K. M. Reddy, N. Y. Graces, M. H. Engelhard, V. Shutthanandan, M. Luo, C. Xu, N. C. Giles, C. Wang, S. Thevuthasan, and A. Punnoose, J. Phys.: Condens. Matter 19, 266203 (2007).

[33] S. K. Misra, S. I. Andronenko, S. Srinivasa Rao, J. Chess, and A. Punnoose, J. Magn. Magn. Mater. 394, 138 (2015).

[34] J. J. Beltràn, C. A. Barrero, and A. Punnoose, J. Solid State Chem. 240, 30 (2016).

[35] C.-W. Liu, S.-J. Chang, S. Brahma, C.-H. Hsiao, F. M. Chang, P. H. Wang, and K.-Y. Lo, J. Appl. Phys. 117, 084315 (2015).

[36] S. Stoll and A. Schweiger, J. Magn. Reson. 178, 42 (2006).

[37] P. Koidl, Phys. Rev. B 15, 2493 (1977).

[38] R. O. Kuzian, A. M. Daré, P. Sati, and R. Hayn, Phys. Rev. B 74, 155201 (2006).

[39] X. Gratens, V. Bindilatti, N. F. Oliveira, Y. Shapira, S. Foner, Z. Golacki, and T. E. Haas, Phys. Rev. B 69, 125209 (2004).

[40] T. Chanier, M. Sargolzaei, I. Opahle, R. Hayn, and K. Koepernik, Phys. Rev. B 73, 134418 (2006).

[41] T. Chanier, F. Virot, and R. Hayn, Phys. Rev. B 79, 205204 (2009). 